\title{
Renditedruck der Finanzmärkte - schwere Zeiten für die Unternehmensethik
}

\author{
BERNHARD EMUNDS ${ }^{*}$
}

\section{Financial Markets Forcing to Profitability - Hard Times for Business Ethics}

The article criticises the recent tendency in German literature to focus on corporations rather than political actors as change agents for ethically sound economic practice. Due to the fact, that capitalistic enterprises primarily try to make profit, they are principally hard to convince to increase human, social and natural capital. In present days, these problems have been exacerbated by the pressure to achieve high returns within a short period of time. This is rooted in a new market-dominated type of financial system in which managers are under strain, as well as in the propagation of the concept of shareholdervalue.

Keywords: Market dominated financial system, shareholder value, demanded rate of return, short-termism, CSR activities as investments

\section{Einstieg: Der umstrittene Stellenwert der Unternehmen bei der ethischen Gestaltung der Wirtschaft}

Welche Rolle kommt den Unternehmen und ihren Vorständen bei der ethisch gebotenen bzw. sinnvollen Veränderung des Wirtschaftens zu? Das Spektrum der Antworten auf diese für die Wirtschafts- und Unternehmensethik zentrale Frage ist in der deutschsprachigen Literatur sehr weit. Die Antworten variieren, je nachdem wie die Autorinnen und Autoren die ethischen Verpflichtungen und die wirtschaftlichen Aufgaben des Managements verstehen und wie sie deren Verhältnis zueinander sehen. Karl Homann und seine Schüler verorten ,die Moral' nicht nur vorrangig in den Rahmenbedingungen, sondern kennen in Bezug auf die Geschäftspolitik eigentlich nur eine Verpflichtung der Manager, nämlich die der Gewinnmaximierung (Homann/BlomeDrees 1992: 38, 51; Homann/Lütge 2005: 91). Diese Aufgabe soll freilich langfristig verstanden werden, damit Unternehmen ihren Einsatz für Regelverbesserungen als eine Chance wahrnehmen können, das eigene Gewinnstreben so zu forcieren, dass zugleich auch die sozialen und ökologischen Anliegen umfassender verwirklicht werden (Pies et al. 2009: 10f.; vgl. Pies 2009). Auf der anderen Seite des Spektrums läuft Peter Ulrich Gefahr, die grundlegende Aufgabe der Vorstände - dafür Sorge zu tragen, dass das Unternehmen am Markt bestehen kann - nicht systematisch zu berücksichtigen und die Manager mit moralischen Verpflichtungen zu überfordern. Unternehmen werden beinahe zu Agenturen für Weltverbesserung stilisiert, wenn Ulrich

\footnotetext{
Prof. Dr. Bernhard Emunds, Oswald von Nell-Breuning-Institut für Wirtschafts- und Gesellschaftsethik der Phil.-Theol. Hochschule Sankt Georgen, Offenbacher Landstraße 224, D-60599 Frankfurt/Main, Tel.: +49-69-6061-230 , Fax: +49-69-6061-559, E-Mail: nbi@sankt-georgen.de, Forschungsschwerpunkte: Grundlagen der Wirtschafts- und der Christlichen Gesellschaftsethik, Theorie und Ethik der Finanzwirtschaft, Ethik der Erwerbsarbeit und des Sozialstaats.
} 
das Management bei grundlegenden Entscheidungen über die Gesamtausrichtung des Unternehmens auffordert, zuerst eine lebensdienliche unternehmerische Wertschöpfungsidee zu suchen: „Am Anfang steht nicht ein strategisches Kalkül, sondern die lebenspraktische Frage: Wofür setzen wir uns ein?" (Ulrich 1998: 432). Und mit seiner „Leitidee deliberativer Unternehmensethik“ (ebd.: 439) die Vorstände größerer Unternehmen darauf verpflichtet, ihre Geschäftsstrategien in ,ergebnisoffenen' StakeholderDialogen zwischen allen Betroffenen-Gruppen zur Disposition zu stellen, bindet er unternehmenspolitische Entscheidungen an langwierige Konsensbildungsprozesse. Dabei scheint er auszublenden, dass die (möglichen) Effizienzvorteile kapitalistischer Marktwirtschaften gegenüber anderen Wirtschaftssystemen gerade auf spezifische Formen der Handlungskoordination zurückgehen: Der Koordination der Handlungen primär über bzw. in Hinblick auf ihre monetären Folgen. Diese können jedoch gerade dann nicht realisiert werden, wenn die Akteure darauf verpflichtet werden, ihre wirtschaftlichen Handlungspläne konsensuell aufeinander abzustimmen. ${ }^{1}$

Eine recht überzeugende mittlere Position vertreten z. B. Horst Steinmann und seine Schüler. Einerseits negieren diese nicht die Gewinnorientierung von Unternehmen und weisen die Aufgabe, das Wirtschaften auf ethische Ziele auszurichten, allen voran den politischen Akteuren zu. Andererseits ist ihnen aber bewusst, dass die rechtliche Ordnung stets auch defizitär ist und deshalb die Vorstände darauf verpflichtet werden müssen, ihre gewinnorientierten unternehmerischen Einzelentscheidungen einer moralischen Prüfung zu unterziehen. ${ }^{2}$ Nicht nur durch die zahlreichen SteinmannSchülerinnen und -Schüler gab es in den letzten Jahren im deutschen Sprachraum immer mehr wirtschafts- und unternehmensethische Veröffentlichungen, die einer solchen mittleren Linie zuzuordnen sind. Dabei fällt allerdings auf, dass sich in Folge der beinahe allgegenwärtigen Globalisierungsdiskurse, aber wohl auch aufgrund der verstärkten Rezeption der anti-etatistischen ,business ethics'-Literatur aus den USA sowie durch die mittlerweile stark von den Unternehmen selbst dominierte CSRBewegung (vgl. Curbach 2009) die Gewichte verschoben haben. Von den nationalstaatlichen Regierungen werden immer weniger, von den Unternehmen, insbesondere von den transnationalen Großkonzernen, werden immer mehr Beiträge zur ethischen Gestaltung des Wirtschaftens erwartet.

Eine fundierte Begründung für diese Akzentverschiebung findet sich z. B. in der Habilitationsschrift des Steinmann-Schülers Andreas Georg Scherer. Für Scherer (2003: 122) schwächt die Globalisierung „das Vermögen des Nationalstaates, der Wirtschaft

1 In seinen Überlegungen zur staatlichen Ordnungspolitik nimmt Ulrich (z. B. 1998: 333) auch auf die Effizienzvorteile der Marktkoordination Bezug. Aber die Aufgabe des Managements, dafür zu sorgen, dass sich das Unternehmen dauerhaft am Markt behauptet, spielt bei Ulrich erst auf der Ebene der Anwendung ethischer Normen (Stichwort ,Zumutbarkeit') eine Rolle. Zu den spezifischen Formen der Koordination wirtschaftlicher Handlungen auf Märkten (,primär über ... ') und in Unternehmen (,primär in Hinblick auf die monetären Folgen') vgl. Emunds (2010: 1847, 18491851).

2 Nach wie vor lesenswert: Steinmann/Löhr (1994: 107-120). Problematisch ist allerdings der Begriff 'Gewinnprinzip' (Gewinnmaximierung als ,Formalziel' des Unternehmens), der in neueren Veröffentlichungen aus dem Umfeld Steinmanns wohl eher gemieden wird. Vgl. z. B. Scherer (2003: 422) "Formalziel der Gewinnerzielung". 
einen Ordnungsrahmen zu geben, der im Großen und Ganzen dafür sorgt, daß die Strategien der Unternehmen mit den Interessen der Gesellschaft verträglich“ sind. Die Unternehmen können nämlich einen Teil ihrer Wertschöpfungsaktivitäten in Länder mit weniger anspruchsvollen Umwelt- und Sozialstandards auslagern oder auch mit der Androhung solcher Verlagerungen die nationalstaatlichen Regierungen unter Druck setzen, gesetzlich bestehende, höhere Anforderungen abzusenken. Aufgrund dieser Schwächung der Regierungen steigt die Bedeutung einer ethischen Selbstverpflichtung der Unternehmen bzw. ihrer Manager, die über die Einhaltung bestehender Gesetze hinausgeht. Von Unternehmen wird deshalb gefordert, sich konstruktiv an der ethisch gebotenen Weiterentwicklung der nationalen und internationalen Regelwerke zu beteiligen und vor allem, da die Steuerungsmöglichkeiten über Gesetze geringer geworden sind, ihr eigenes Geschäftsgebaren ethisch auszurichten (Scherer 2003: 428-433).

Der vorliegende Beitrag ist als Kritik an der aktuellen Tendenz in der Wirtschafts- und Unternehmensethik konzipiert, ethisch gebotene Veränderungen des Wirtschaftens weniger auf dem ,Umweg' über das politische System als durch ethische Beratung der Unternehmensführung anzuzielen. Dem Beitrag liegt hierbei die am Nell-BreuningInstitut konzipierten Politischen Wirtschaftsethik (vgl. Hengsbach 1991, 1992, 1995; Emunds 1995, 2009b, 2010). zu Grunde. In dieser wird die Wirtschaft zuerst als Handlungsbereich der Gesellschaft begriffen und diese primär als ein politisches Gemeinwesen, dessen Bürgerinnen und Bürger in demokratischen Prozessen die Institutionen des Zusammenlebens nach allgemein oder weithin geteilten Vorstellungen einer guten Ordnung zu gestalten suchen. Aufgrund ihrer Wohlstandsziele unterstellen diese einen Großteil der wirtschaftlichen Handlungen einer marktwirtschaftlichen Logik, versuchen aber zugleich die Institutionen der Wirtschaft so zu gestalten, dass sie nicht nur ihren funktionalen Erwartungen, sondern auch ihren weitergehenden normativen Ansprüche entsprechen. Die Politische Wirtschaftsethik geht davon aus, dass in kapitalistischen Marktwirtschaften die Vorstände von Unternehmen faktisch zuerst einmal die Aufgabe haben, dafür zu sorgen, dass sich ihr Unternehmen am Markt behaupten kann, damit die Wertschöpfung und die Entstehung von Einkommen für Kapitalgeber und Arbeitnehmer dauerhaft gesichert sind. Aufgrund dieser Einschränkungen des unternehmerischen Handlungsspielraums wird die (supra)staatliche Steuerung des Wirtschaftens durch rechtliche Normen und deren Kontrolle durch vitale politische Öffentlichkeit als wichtigster Ansatzpunkt für ethisch gebotene bzw. sinnvolle Veränderungen des Wirtschaftens begriffen. Trotzdem können und sollen sozial und ökologisch verantwortlich geführte Unternehmen eine wichtige Rolle spielen: als Innovatoren, die von sich aus ethisch sinnvolle Veränderungen der wirtschaftlichen Wertschöpfung vorantreiben, und als kollektive politische Akteure, die konstruktiv zu einer Weiterentwicklung der Rahmenbedingungen beitragen. ${ }^{3}$ Dabei

3 Vor allem in Anschluss an Enderle (z. B. 1988: 55-58) wird deshalb zu Recht in der Wirtschaftsethik die Dichotomie zwischen Mikro- und Makro-Ebene durch Berücksichtigung der MesoEbene kollektiver Akteure (einschließlich der Unternehmen als wirtschaftliche Organisationen) aufgebrochen. Aus moraltheoretischer Sicht ist es jedoch problematisch, Unternehmen den Status moralischer Handlungssubjekte zuzusprechen und sie als ,Subjekte‘ bzw. ,Träger moralischer Verantwortung' zu bezeichnen. 
werden die Unternehmen auch intern nicht als ,politikfreie’ Räume begriffen, sondern als umstrittene Teilöffentlichkeiten bzw. als Arenen für Interessenkonflikte und Konsensbildungsprozesse. Die Entscheidungsträger in den Unternehmen sieht die Politische Wirtschaftsethik mit legitimen ethischen Ansprüchen höchst unterschiedlicher Verbindlichkeit konfrontiert - bis hin zu strikt einzuhaltenden moralischen Unterlassungsnormen, wie der, keinem Produktionsprozesse zu organisieren, in denen Arbeitnehmer unter menschenunwürdigen Bedingungen arbeiten müssen. ${ }^{4}$

Der Perspektive einer Politischen Wirtschaftsethik entsprechend soll im Folgenden die starke Betonung der Unternehmen als Träger ethisch sinnvoller Veränderungen des Wirtschaftens kritisiert werden, die für die neuere wirtschafts- und unternehmensethische Literatur im deutschen Sprachraum charakteristisch ist. Bei dieser Sicht werden die Möglichkeiten von Unternehmensvorständen, soziale und ökologische Zielsetzungen in ihre geschäftsstrategischen Entscheidungen zu integrieren, zu optimistisch eingeschätzt. Dieser Kritikpunkt wird im folgenden Abschnitt (2.) zunächst grundsätzlich, d. h. mit Blick auf kapitalistische Unternehmen überhaupt, skizziert. Ohne eine konsequente Regulierung mit Hilfe staatlichen Rechts hat das Bestreben von Unternehmensleitungen, Kosten zu senken und Aufwand zu externalisieren, gravierende soziale und ökologische Folgen. Vorgeschlagen wird, diesen Zusammenhang als einen für kapitalistische Marktwirtschaften typischen, inneren Widerspruch der Vermögensentwicklung auf den Begriff zu bringen. Da die Rendite auf das Kapital eines Unternehmens zumeist nur zu einem kleineren Teil ausgezahlt wird, sollte davon ausgegangen werden können, dass mit einer Steigerung der Rendite auch eine Beschleunigung der Vermögensakkumulation einhergeht. Hohe Renditen sind jedoch häufig mit einem forcierten Verschleiß bzw. mit einem reduzierten Erhaltungsaufwand verbunden, so dass das Vermögen als Grundlage künftiger Einkommen bzw. Lebenschancen erodiert. In dem zentralen Abschnitt des Beitrags (3.) soll dann herausgearbeitet werden, dass der grundlegende Strukturwandel der Finanzwirtschaft in den letzten zwanzig Jahren diesen inneren Widerspruch verschärft hat. Der Beitrag endet mit einigen Schlussfolgerungen für die wirtschafts- und unternehmensethische Debatte (4).

\section{Aufbau und Erosion verschiedener Vermögensformen in der kapitalistischen Marktwirtschaft ${ }^{5}$}

In der Ökonomie wird Vermögen als etwas definiert, mit dem der Anspruch auf (oder zumindest doch die berechtigte Erwartung von) Einkommenszahlungen in der Zukunft verbunden ist. Der Wert des Vermögens ergibt sich aus der Bewertung dieses künftigen Einkommens. Besser noch als durch den Blick auf aktuelle Einkommensrelationen kann man durch eine Analyse der Vermögensverteilung und ihrer Veränderungen tiefgreifende und dauerhafte Entwicklungen identifizieren, welchen gesellschaftlichen Gruppen welche wirtschaftlichen Möglichkeiten eröffnet werden oder verschlos-

$4 \quad$ Vgl. - mit Blick auf Sozialstandards - Sachverständigengruppe 2008: 41-51. Für eine Unterscheidung zwischen verschiedenen Verpflichtungsgraden unternehmensethischer Normen plädieren z. B. auch Aßländer/Brink 2008: 111-116.

5 In der folgenden Skizze greife ich u. a. Anregungen auf, die ich Friedhelm Hengsbach SJ und Ottmar Edenhofer verdanke. 
sen bleiben. ${ }^{6}$ Dabei sollte jedoch der Begriff des Vermögens nicht auf Betriebs-, Geldund Immobilienvermögen beschränkt, sondern sehr weit gefasst werden.

Einzubeziehen ist zum einen das Arbeitsvermögen, also die Aussicht der Erwerbstätigen, dadurch Einkommen zu erzielen, dass sie die Verfügung über ihre Arbeitskraft einschließlich der Möglichkeit, die von ihnen erworbenen besonderen Fähigkeiten zu nutzen, an andere verkaufen. ${ }^{7}$ Zum anderen sind, wenn die gesellschaftliche Entwicklung anhand von Veränderungen der Vermögensbestände untersucht werden soll, auch diejenigen Vermögensformen zu berücksichtigen, die nicht mit monetärem Einkommen verbunden sind. Vermögen steht dann nicht nur für erwartete Einnahmen in der Zukunft, sondern für künftige Lebenschancen von Menschen überhaupt. Vor allem nach dieser Weitung des Vermögensbegriffs ist es sinnvoll, neben dem Kapital von Unternehmen (im Sinne des Besitzes an Produktionsmitteln), dem Geld- und Immobilienvermögen sowie dem Arbeitsvermögen, eben auch von Gesellschafts- und Naturvermögen zu sprechen und deren Entwicklung in die Gesellschaftsanalyse und wirtschaftsethische Reflexion einzubeziehen. Gesellschaftsvermögen steht dabei für die Möglichkeiten der Menschen, sich in Beziehungsnetzen zu entfalten und am gesellschaftlichen Leben - vor allem am demokratischen Prozess der Meinungsbildung zu beteiligen. Und der Begriff ,Naturvermögen' bezeichnet eine natürliche Umwelt, deren Ressourcen Menschen nicht nur nutzen, um Güter anbieten zu können, sondern in der sie auch gerne leben und deren Schönheit sie genießen. ${ }^{8}$ Bei einem derart weiten Vermögensbegriff kann der Erhalt und die Bildung von Vermögen als eine zentrale Aufgabe der Gesellschaftspolitik angesehen werden. Sie soll durch die Festlegung geeigneter Regeln und durch andere Interventionen die ,Weichen' so ,stellen', dass sich aus dem Handeln der gesellschaftlichen Akteure eine dauerhafte und ausgewogene Mehrung der verschiedenen Vermögensformen ergibt, die für alle Gesellschaftsglieder vorteilhaft ist. ${ }^{9}$ Eine solche Vermögensbildung ist nur möglich, wenn die Gesellschaft den Aufwand zur Reproduktion bzw. zum Ersatz ,verschlissenen' Vermögens, zum Schutz bestimmter Vermögensformen vor Übernutzung und zum Aufbau zusätzlichen Vermögens nicht scheut.

Für die kapitalistische Marktwirtschaft typisch ist zum einen der Versuch, die verschiedenen Formen solcher Aufwendungen monetär zu bewerten. Zumeist kann eine Gesellschaft mit kapitalistischem Wirtschaftssystem nur dann sicherstellen, dass der Aufwand zum Erhalt bzw. zur Mehrung einer bestimmten Vermögensform auch wirklich kontinuierlich erbracht wird, wenn sie in Entsprechung zu diesem Aufwand ein

6 Die katholische sozialethische Tradition hatte ein Gespür für die zentrale Bedeutung der Vermögensverteilung. Das zeigt ihre Forderung, die abhängig Beschäftigten durch Vermögensbildung am Produktionsmittelbesitz zu beteiligen und auch auf diesem Weg die kapitalistischen Machtverhältnisse langfristig ,umzubiegen' (vgl. z. B. Nell-Breuning 1954, 1986: 147-192). Leider wurde dieser Reformpfad zum Eigenheim-Sparen banalisiert und durch das 312 Mark-Gesetz und seine Nachfolger verniedlicht.

7 Zum Begriff des Arbeitsvermögens vgl. Hengsbach/Möhring-Hesse 2002 mit Bezug auf Cohen 2001.

$8 \quad$ Weiterführende Überlegungen zum Begriff des Naturkapitals bei Ott/Döring 2008: 179-231.

$9 \quad$ Bei einer Entfaltung dieser wirtschaftsethischen Perspektive sind die Aspekte „dauerhaft“, „ausgewogene Mehrung verschiedener Vermögensformen“ und „für alle Gesellschaftsglieder vorteilhaft" natürlich zu präzisieren. 
System von Zahlungen durchsetzt, welches die Gesellschaftsglieder zu erbringen haben: vor allem Arbeitseinkommen, Renten für die Besitzer natürlicher Produktionsmittel sowie Steuern und Abgaben (Binswanger 2006: 208f., 266-284). Wesentlich für das bestehende Wirtschaftssystem ist zum anderen, dass eine Vielzahl der Güter durch kapitalistische Unternehmen, also durch Organisationen, die primär gewinnorientiert geführt werden und sich der Arbeitskraft von abhängig Beschäftigten bedienen, bereitgestellt wird. Dabei bedeutet ,primär gewinnorientiert', dass die Unternehmensleitung bei der Organisation der Wertschöpfung vorrangig das Ziel hoher Einkommen für die Eigenkapitalgeber verfolgt. ${ }^{10}$ Oder in Vermögenskategorien ausgedrückt: Die unternehmerische Wertschöpfung wird vor allem so organisiert, dass das Eigenkapital wächst. In der theoretischen Beschreibung von Unternehmen, aber vielfach auch in der Realität steht diese Ausrichtung so eindeutig im Vordergrund, dass häufig die Gewinnmaximierung als die Zielsetzung kapitalistischer Unternehmen begriffen wird.

Aufgrund der Dominanz des Gewinnziels bei der Organisation der unternehmerischen Wertschöpfung ist die Bereitstellung von Gütern durch kapitalistische Unternehmen durch einen inneren Widerspruch gekennzeichnet. Einerseits ist unternehmerische Wertschöpfung nur möglich, wenn die Unternehmen das Arbeits-, Gesellschafts- und Naturvermögen nutzen können. Auf die Dauer können die Unternehmensvorstände die Gewinne deshalb nur dann steigern und insofern das Eigenkapital mehren, wenn auch diese Vermögensformen langfristig zuverlässig zur Verfügung stehen. Andererseits erfordert der Erhalt bzw. der Ausbau dieser Vermögensformen Aufwendungen. Müssen die Unternehmen diese selber tragen, treten diese in ihrer Kosten-Leistungsrechnung sofort als Kosten in Erscheinung, die ihre Gewinne schmälern. Deshalb bemühen sich kapitalistische Unternehmungen darum, einen möglichst großen Teil der Aufwendungen an die Gesellschaft weiterzugeben, also Kosten zu externalisieren, und dort, wo sie die Kosten doch selbst tragen müssen, diese möglichst gering zu halten. Der Widerspruch besteht nun genau darin, dass die zur kurzfristigen Gewinnsteigerung sinnvollen Bemühungen der Unternehmen, Kosten zu reduzieren, in vielen Fällen bedeuten, dass die Aufwendungen für den Erhalt bzw. Aufbau des Arbeits-, Gesellschafts- und Naturvermögens sinken. Dies beeinträchtigt die Entwicklung der Entfaltungs- und Beteiligungsmöglichkeiten aller Bürgerinnen und Bürger - und zugleich auch die künftigen Chancen der Unternehmen, Gewinne zu erzielen und darüber das unternehmerische Eigenkapital zu vergrößern. Reduziert wird also die gesamtgesellschaftliche Vermögensbildung und damit auch die ,Pflege' derjenigen Vermögensformen, deren gute Entwicklung langfristig eine wichtige Voraussetzung für den Erfolg von Unternehmen darstellt..11

10 Dabei kann hier offen bleiben, ob es bei den Eigenkapitalgebern um die Eigentümer geht, die tatsächlich für den Geldvorschuss gesorgt haben, ohne den die unternehmerische Wertschöpfung nicht möglich wäre, oder um Aktionäre.

11 Damit ist natürlich nicht ausgesagt, dass der Aufbau verschiedener Vermögensformen, den die Bürgerinnen und Bürger einer Gesellschaft wünschen, einfach identisch sei mit den Zielen von Unternehmen, die für ihre Wertschöpfung relevanten Teile des Arbeits-, Gesellschafts- und Naturvermögens zu erhalten bzw. zu mehren. 
Dieser Widerspruch ist für das kapitalistische Wirtschaftssystem insgesamt kennzeichnend, ${ }^{12}$ er kann durch eine soziale und ökologische Bändigung des Kapitalismus politisch begrenzt werden. In den letzten zwanzig Jahren hat sich der Widerspruch jedoch massiv verschärft. Das liegt zum einen an dem gestiegenen Einfluss des marktradikalen Wirtschaftsliberalismus, der die Bemühungen, den Kapitalismus mit Hilfe des Rechts sozial (und ökologisch) zu bändigen, erlahmen ließ, zum anderen, wie der folgende Abschnitt zeigen wird, an einer grundlegenden Transformation der Finanzwirtschaft.

\section{Der Renditedruck der neuen Finanzwirtschaft}

Bei dem inneren Widerspruch der Vermögensakkumulation, der im vorhergegangenen Abschnitt skizziert wurde, geht es darum, dass das Ziel des Vermögensaufbaus mit Hilfe einer betriebswirtschaftlichen Logik verfolgt wird, die in der kurzen Frist mit der Vermeidung bzw. dem Abwälzen von Kosten ein Element beinhaltet, das sich, wenn es besonders stark gewichtet und konsequent verfolgt wird, langfristig auf das Verfolgen dieses Ziels negativ auswirkt. Im Vordergrund stehen dabei eine gesellschaftliche (bzw. globale) Perspektive, bei der es um die vernachlässigte ,Pflege’ der verschiedenen Vermögensformen geht, und eine gesamtwirtschaftliche Perspektive, der gemäß sich forcierte Kostensenkungsstrategien auf die Dauer ${ }^{13}$ negativ auf die Akkumulation des Eigenkapitals aller Unternehmen auswirken können. Die Aufgabe, mögliche Inkongruenzen zwischen der kurzen und der langen Frist herauszustellen, ist - nun direkt auf der betriebswirtschaftlichen Ebene - auch in der Unternehmensethik immer präsent. Schließlich bemühen sich viele Unternehmensethiker nicht nur darum, die Handlungsmotive der Unternehmensvorstände mit Hilfe ethischer Argumente zu beeinflussen, sondern sie versuchen zugleich, den Managern bzw. Führungskräften nahe zu bringen, dass sich sozial und ökologisch verantwortliche Unternehmensstrategien langfristig auf die Gewinnentwicklung des Unternehmens positiv auswirken werden. Die Wahl solcher Strategien wird u. a. bei Homann und seinen Schülern als Investition - und damit als Aufbau von Vermögen - begriffen, weil die Ausgaben für eine bessere Reputation des Unternehmens bei den potentiellen Käufern oder für eine höhere Motivation der Mitarbeiter sofort anfallen und erst deutlich später zu höheren Einnahmen führen können. ${ }^{14}$ Allerdings stellt sich die Frage, wie weit diese unternehmensethische Strategie, die ethisch gebotenen oder wünschenswerten Geschäftsstrategien als langfristig erfolgreicher herauszustellen, in der Gegenwart trägt. Wie im Folgenden herausgearbeitet werden soll, haben nämlich die Kapitalgeber zumindest der großen Konzerne heute nicht nur sehr hohe Gewinnansprüche (3.3), sondern lassen den

$12 \mathrm{Ob}$ der Widerspruch zwischen kurzfristigen betriebswirtschaftlichen Zielen und langfristigen Erfordernissen der Vermögensakkumulation - ggf. in abgewandelter Form - auch in anderen Wirtschaftssystemen prägend wäre, kann hier offen bleiben.

13 Nebenbei sei erwähnt, dass es einen vergleichbaren Zusammenhang auch in der kurzen Frist gibt, weil Kostensenkungen Einkommensausfälle bedeuten, mit denen ein Rückgang der Güternachfrage verbunden sein kann.

14 Z. B. Homann 2001; zur Diskussion über die Funktionalisierung bzw. Instrumentalisierung der Moral vgl. u. a. Homann-Blome-Drees 1992: 142; Ulrich 1998: 416-421; Wieland 2005: 60-69, 7881. 
Managern auch nur wenig Zeit, diese zu erfüllen (3.2). Begründet ist diese neue Konstellation in der Herausbildung einer neuen Form der Finanzwirtschaft (3.1).

\subsection{Die neue kapitalmarktdominierte Finanzwirtschaft}

Die Finanzsysteme der Industrieländer lassen sich in den ersten drei Jahrzehnten nach dem Zweiten Weltkrieg zwei Typen zuordnen (vgl. u. a. Allen/Gale 2000). In Japan, Deutschland, aber auch in anderen Ländern des westlichen Kontinentaleuropa spielten die Geschäftsbanken mit ihrem Kredit- und Einlagengeschäft bei der Unternehmensfinanzierung und der Ersparnisbildung der privaten Haushalte die alles beherrschende Rolle. Da es ihnen zudem erlaubt ist, zahlreiche andere Finanzdienstleistungen anzubieten sowie Aktien zu besitzen und da sie eine wichtige Rolle bei der Kontrolle von Unternehmen spielen (Universalbanken), können die damaligen Finanzsysteme dieser Länder als rein bankendominiert charakterisiert werden. Ihnen standen im anglo-amerikanischen Raum marktdominierte Finanzsysteme gegenüber. Hier waren bereits zu dieser Zeit Kapitalmärkte, also die Märkte für Aktien und Rentenpapiere (langfristige handelbare Schuldtitel), für die Finanzierung der Unternehmen und für die Vermögensbildung der privaten Haushalte sehr bedeutsam. Dabei war es den Kreditinstituten in den USA strengstens verboten, Unternehmensanteile zu erwerben oder über das Kredit- und Einlagengeschäft hinaus ein breiteres Spektrum von Finanzdienstleistungen anzubieten. Statt der Geschäftsbanken waren für die Begleitung von Wertpapiergeschäften, die Finanzierung von Unternehmen über den Kapitalmarkt und die professionelle Vermögensanlage andere, entsprechend spezialisierte, Finanzinstitute zuständig, vor allem die sogenannten Investmentbanken (,Trennbankensystem').

Auch wenn nationale Besonderheiten, z. B. die unterschiedlich starke Bedeutung von Geschäftsbanken, nicht völlig verschwunden sind, hat sich seit den 1980er Jahren in allen Industrieländern allmählich eine neue, vergleichsweise einheitliche, Konstellation der Finanzwirtschaft entwickelt, die hier als neue kapitalmarktdominierte Finanzwirtschaft bezeichnet wird. Ausgangspunkt für diese Veränderungen war der Wandel des Wertpapierbesitzes in den marktdominierten Finanzsystemen der USA und Großbritanniens. Waren in diesen Ländern die privaten Haushalte früher selbst direkt die Käufer und Besitzer der Wertpapiere, so schalteten sie jetzt immer häufiger institutionelle Investoren, insbesondere Investment- und Pensionsfonds, zwischen (vgl. z. B. Shiller 2000: 52f.; Windolf 2008: 518f.). Seitdem kaufen die Haushalte mit ihren Ersparnissen vor allem Anteile der Fondsgesellschaften und überlassen es den Mitarbeitern der Fonds, ein Portfolio an Vermögenswerten professionell zusammenzustellen. Vielfach haben die professionellen Anleger sogar den Auftrag, das Portfolio kontinuierlich zu ,optimieren', also ständig Vermögenswerte zu verkaufen und neu zu kaufen (,aktiv gemanagte Fonds'). Zugleich erhöhten die Fondsverwalter als Shareholder den Druck auf die Vorstände der Konzerne durch Einführung neuer Strukturen der Unternehmenssteuerung (,corporate governance'), Verkauf oder Zukauf von Unternehmensteilen, höhere Ausschüttungen, stärkere Verschuldung, Veränderungen im Kerngeschäft oder auch nur durch Professionalisierung der ,Kapitalmarktkommunikation' immer wieder für einen Anstieg des Aktienkurses zu sorgen. In der Folge kam es zu einer schnellen Expansion jener Teilbereiche der Finanzwirtschaft, die auf Transakti- 
onen und Finanzdienstleistungen mit Wertpapieren und Derivaten spezialisiert sind und zum Investmentbanking gezählt werden. Die Investmentbanken profitierten nicht nur von einem starken Anstieg der Volumina aller Finanztransaktionen, sondern zugleich erfanden ihre Mitarbeiter ständig neue Finanztitel. Solche Finanzinnovationen erschlossen scheinbar den Kunden, insbesondere den Geschäftsbanken und Fonds immer bessere Möglichkeiten des Risikomanagements. Oder sie boten den Finanzinstituten immer neue Möglichkeiten, jene staatlichen Vorschriften zu umgehen, die sie bei der Expansion der Geschäfte und der damit verbundenen Akkumulation von Risiken begrenzen sollten. Etwa in der gleichen Zeit, also seit den 1980er Jahren, beschlossen die US-amerikanischen Politiker mehrere Gesetze, welche das Trennbankenprinzip, also die strikte Trennung zwischen den Geschäftsbanken mit dem Kreditund Einlagengeschäft und den Investmentbanken mit dem Wertpapiergeschäft, Schritt für Schritt aufweichten. Das Ergebnis dieser Veränderungen war, dass in den angloamerikanischen Finanzsystemen ein hochdynamisches Investmentbanking-Segment entstand, an dem sich, besonders bei der Finanzierung der Vermögensumschichtungen, die Geschäftsbanken rege beteiligten.

Die gleiche Gesamtkonstellation der Finanzwirtschaft setzte sich in etwa auch in Japan sowie mit der Zeit im westlichen Kontinentaleuropa und in einigen Schwellenund Transformationsländern durch. Teils bereits in den $1980 \mathrm{er}$, vor allem aber in den 1990er Jahren kam es in diesen Ländern zu einem schnellen Wachstum der bis dahin nur kümmerlich entwickelten Wertpapiermärkte und - nach anglo-amerikanischem Vorbild - zur Entwicklung und zügigen Expansion des Investmentbankings. Es entstanden große Fondsgesellschaften und die Märkte für Wertpapiere (inkl. Derivaten) sowie - in einigen Ländern - für Immobilien belebten sich (vgl. z. B. Shiller 2000: 56f.; Dembinski 2008: 115-125). Großbanken und Großunternehmen, die etwas auf sich hielten, begannen, ihr Risikomanagement durch den Einsatz immer neuerer und komplizierterer Finanztitel und Risikosteuerungsmodelle kontinuierlich zu ,optimieren'. Zugleich leiteten sie unter dem Schlagwort ,Shareholder Value' diverse kosmetische und reale Veränderungen ein, um den eigenen Aktienkurs immer weiter in die Höhe zu treiben. Da diese Länder das Spektrum der Geschäftsfelder ihrer Banken schon immer nur unwesentlich eingeschränkt hatten (,Universalbanken') und solche Beschränkungen jetzt auch nicht neu einführten, war hier von Anfang an das Segment der Wertpapiergeschäfte bzw. des Investmentbanking eng mit den Geschäftsbanken verbunden.

\subsection{Kurzfristiger Renditedruck}

Aus Sicht der Unternehmen in der Bundesrepublik stellt sich der Wandel der Finanzwirtschaft zuerst einmal als partieller Verlust jenes ,geduldigen Kapitals“ (Streeck 1999: 24; Lane 2000: 210) dar, das für eine Orientierung der Vorstände am langfristigen Unternehmenserfolg gute finanzwirtschaftliche Voraussetzungen bot.

Traditionell sind in Deutschland die industriellen Beziehungen auf allen Ebenen vergleichsweise stark durch die Bereitschaft zu einer Kooperation zum Vorteil aller Beteiligten oder zu einer Befriedung von Konflikten durch einen fairen Kompromiss geprägt. Typisch ist die langfristige Bindung zwischen den Unternehmen und ihrer (Kern-),Belegschaft', mit anderen Worten, die im internationalen Vergleich auffallend 
hohe Verpflichtungskraft in den unvollständigen Verträgen (Richter/Furubotn 2003: 269-276) zwischen Arbeitgebern und Arbeitnehmern. Dies ermöglicht ein vergleichsweise hohes Niveau der betriebsspezifischen Qualifikation und fördert die Motivation der qualifizierten Mitarbeiter, einen hochwertigen Beitrag zum Erfolg des Betriebs zu leisten. Unter anderem ist aufgrund der starken Position der Arbeitnehmervertreter in der Betriebsverfassung das Vertrauen der Mitarbeiter vergleichsweise hoch, dass das Management auch in Zukunft unternehmerische Herausforderungen nach Möglichkeit durch Strategien im Interesse aller Beteiligten beantworten oder zumindest die für sie schmerzhaften Veränderungsprozesse durch faire Kompromisse abfedern wird. Der Schutz vor einer Übervorteilung ermutigt die Beschäftigten dazu, eine hohe betriebsspezifische Qualifikation aufzubauen, die im Falle einer Kündigung ja weitgehend entwertet würde. Und die starke Bindung der Beschäftigten an ,ihren' Betrieb bzw. an ,ihr' Unternehmen bietet auch den Firmen die Sicherheit, dass sich hohe Ausgaben für die Qualifizierung ihrer Mitarbeiter noch auszahlen werden (vgl. Soskice 1999: 214; Hackethal/Schmidt 2000: 76-77). Mit ihrer motivierten Facharbeiterschaft gelang es der deutschen Industrie über Jahrzehnte hinweg besonders gut, die Vorteile flexibler, computergesteuerter Fertigungssysteme für eine Steigerung der Qualität und Variantenvielfalt ihrer Produkte zu nutzen. Das Ergebnis war und ist z. T. bis heute eine erfolgreiche Verbindung der Massenproduktion qualitativ hoch stehender Grundelemente oder -typen mit der Produktion kleiner Chargen kundenspezifisch zugeschnittener Produkte (,diversifizierte Qualitätsproduktion'; vgl. Streeck 1992: 4-6). Kurzum, die langfristige Interessenkonvergenz zwischen Arbeitgebern und Arbeitnehmern, dass sich ihr Unternehmen am Markt behaupten möge, konnte in Deutschland das Handeln der Akteure vergleichsweise stark prägen. Das spiegelte sich nicht nur im Selbstverständnis vieler Betriebsräte als Co-Manager, sondern auch in einem vergleichsweise langfristigen Planungshorizont wider.

Finanzwirtschaftlich wurde der langfristige Planungshorizont deutscher Unternehmen durch das ,geduldige Kapital' abgesichert. Konturen gewinnt dieser Begriff, wenn man sich als Kontrastfolie den, vor allem im US-Finanzsystem der 1980er Jahre funktionsfähigen, Markt für Unternehmenskontrolle verdeutlicht. Ein Aktienmarkt fungiert dann als ,market for corporate control' und damit als ein Mechanismus, der das Management einer börsennotierten Aktiengesellschaft auf das Aktionärsinteresse an möglichst hohen Aktienkursen verpflichtet, wenn die Manager bei einem niedrigen Aktienkurs ihres Unternehmens mit der Gefahr rechnen müssen, durch eine feindliche Übernahme ihre gut dotierten Posten zu verlieren. In den 1980er Jahren war diese Gefahr für viele Manager US-amerikanischer Großunternehmen real, weil die Aktien ihrer Unternehmen vielfach vollständig in Streubesitz waren und weil ihnen selbst damals kaum Gegenstrategien (,poison pills') zur Verfügung standen, um drohende feindliche Übernahmen abzuwenden. Anders als im US-Finanzsystem der Gegenwart besaßen die Pensionsfonds damals kaum wirklich große Aktienpakete der Unternehmen, die sie dauerhaft an das jeweilige Unternehmen gebunden hätten. Während heute viele Fonds, sofern sie Großaktionäre sind, die Geschäftspolitik der Unternehmen zu beeinflussen suchen (,Kontrolle'), stand damals bei den Strategien der Fonds ausschließlich die Möglichkeit im Vordergrund, die Aktien schnell und reibungslos verkaufen und das Geld in, derzeit renditeträchtiger erscheinende, Alternativen anlegen zu können (,Liquidität'). Sowohl die mangelnde Konzentration des Aktienbesitzes als 
auch das vorrangige Interesse des breit gestreuten Aktionärpublikums an Liquidität statt an Kontrolle führten zu einer hohen Umschlaghäufigkeit der ausgegebenen Aktien (u. a. Windolf 2008: 527), mit der auch das Drohpotential feindlicher Übernahmen erheblich stieg (vgl. Bolton/Thadden 1998). Um feindliche Übernahmen unwahrscheinlich zu machen, mussten die Manager zügig für einen Anstieg des Aktienkurses sorgen. Sie mussten Geschäftsstrategien wählen (und offiziell bekannt geben), die sich schnell in höheren Gewinnen niederschlagen und von den Analysten als besonders gewinnträchtig eingestuft wurden.

Anders als das ,ungeduldige' Finanzkapital auf dem Markt für Unternehmenskontrolle drängten die Anteilseigner deutscher Unternehmen bis Mitte der 1990er Jahre die von ihnen kontrollierten Manager nicht zu schnellen Renditesteigerungen. Bei großen Kapitalgesellschaften ermöglichte das häufig als ,Deutschland AG' apostrophierte Netzwerk, in das die Großbanken und die meisten größeren Unternehmen eingebunden waren (vgl. Adams 1994; Windolf/Beyer 1995), eine Langfristorientierung. Dieses Netz wurde zum einen durch Kapitalbeteiligung, d. h. den Aktienbesitz von Kapitalgesellschaften an Kapitalgesellschaften, gebildet. Da die unternehmerischen Großaktionäre einer AG vielfach ihre eigenen Vorstandsmitglieder in den Aufsichtsrat dieser Kapitalgesellschaft entsandten, entstand zum anderen ein Personen-Netzwerk der Aufsichtsratsbeziehungen. In dessen Zentrum bildete sich durch die regelmäßigen Sitzungen der Aufsichtsräte, an denen die Beteiligten mal als Kontrollierte, mal als Kontrollierende teilnahmen, ein Club heraus, der durch engen Zusammenhalt gekennzeichnet war und seine Machtposition gemeinsam nach außen absicherte (Windolf/Beyer 1995: 22-25). Das Netzwerk sicherte die Langfristorientierung deutscher Großunternehmen, weil es deren Manager vor der Gefahr feindlicher Übernahmen abschirmte.

Die hohe Bereitschaft deutscher Unternehmen zu Investitionsprojekten, die sich erst langfristig auszahlen würden, wurde zudem durch deren Hausbankbeziehungen abgesichert. Als Hausbank eines Unternehmens wird ein Kreditinstitut bezeichnet, dem einerseits das Unternehmen einen exklusiven und schnellen Zugang zu unternehmensinternen Informationen eingeräumt hat, das andererseits das Unternehmen gegen Zahlungsschwierigkeiten absichert, in dem es ihm in Zukunft Kredite zu annehmbaren Konditionen auch bei einer begrenzten Verschlechterung der Kreditwürdigkeit in Aussicht stellt (vgl. Elsas/Krahnen 1998). Diese Absicherung reduziert das Risiko eines Bankrotts und damit aus Managersicht auch das Risiko, den Vorstandsposten zu verlieren, welches bei Projekten, die sich voraussichtlich erst nach einer langen Anlaufzeit auszahlen werden, besonders hoch ist. Auf diese Weise stärken Hausbankbeziehungen die Bereitschaft der Manager, auch Projekte in Angriff zu nehmen, die sich nur langfristig auszahlen (vgl. u. a. Dewatripont/Maskin 1995). Seit Ende der 1990er Jahre sind es vor allem die Sparkassen und Kreditgenossenschaften, welche die Hausbankbeziehungen zu Kleinen und Mittleren Unternehmen (KMU) beibehalten und neue aufbauen.

Mit der Transformation des deutschen Finanzsystems seit Mitte der 1990er Jahre, also durch den Bedeutungsgewinn der Wertpapiermärkte und den Aufstieg der kapitalmarktbezogenen Teilbranchen der Finanzwirtschaft, ist die Gefahr verbunden, dass die deutschen Unternehmen ihr ,geduldiges Kapital' und damit das finanzwirtschaftli- 
che Rückgrat ihrer Langfristorientierung verlieren. Bis zum Ausbruch der globalen Finanzkrise standen vor allem die Manager dreier Gruppen deutscher Unternehmen eindeutig unter dem finanzwirtschaftlichen Druck, ihre Gewinne schnell steigern zu müssen. Das gilt erstens für die Vorstände einzelner Großunternehmen, die - nicht nur verbal, sondern auch real - auf eine kapitalmarktorientierte Geschäftsführung gesetzt und deshalb dafür gesorgt hatten, dass die Aktien ihres Unternehmens zu mindestens zu 75\% in Streubesitz kamen. Auf diese Weise haben sich diese der Gefahr feindlicher Übernahmen ausgesetzt und damit letztlich selbst unter Druck gesetzt, durch schnelle Gewinnsteigerungen die Gefahr feindlicher Übernahmen abwenden zu müssen. Zweitens ist jener - noch vergleichsweise kleine - Teil der deutschen KMU einem kurzfristigen Gewinndruck ausgeliefert, der vorübergehend von Finanzinvestoren übernommen wurde. Drittens stehen diejenigen KMU oder (ehemaligen) Töchter großer Konzerne unter einem massivem Kurzfristdruck, bei denen durch Übernahmen und erneute Verkäufe die Eigentümer schnell wechseln. Die Manager dieser Gruppen von Unternehmen können die hohen Erwartungen der Analysten und Fondmanager oder der vorübergehenden Besitzer auf eine schnelle Gewinnsteigerung nur „bei Strafe des Untergangs“" (Marx 1964: 255) - hier: bei wahrscheinlichem Verlust ihres Vorstandsposten - enttäuschen. Auf diese Weise wird ihnen der lange Atem abgeschnitten, den sie benötigen, um Investitionen zu tätigen, die sich erst nach einigen Jahren auszahlen werden. Ihre Geschäftspolitik wird kurzatmig.

Der Anstieg der Kurzatmigkeit bei der Gewinnorientierung deutscher Unternehmen ist aber nicht auf die drei beschriebenen Gruppen beschränkt. Vielmehr hat mit der Modernisierung des Finanzsystems zugleich auch ein neues Leitbild unternehmerischen Handelns Fuß gefasst, in dem die Manager nicht mehr aufgefordert werden, die divergierenden Interessen von Eigen- und Fremdkapitalgebern sowie von einflussreichen Arbeitnehmergruppen zum Ausgleich zu bringen, sondern einzig und allein dem Aktionärsinteresse an einem möglichst hohen Aktienkurs verpflichtet sind (vgl. u. a. Kuhner 2004). Um den Shareholder Value zu steigern sind sie, im Rahmen des ,Economic-Value-Added'(EVA®)-Konzepts, dazu angehalten, die Tochterunternehmen und Abteilungen zu Profit-Center umzubauen, die alle ein hochgestecktes Renditeziel zügig erreichen sollen. Dieses Leitbild unternehmerischen Handelns ist nicht nur bei den Analysten und den professionellen Anlegern in den großen Investment- und Pensionsfonds sowie bei Unternehmensberatern weit verbreitet, sondern hat sich allmählich auch bei vielen Vorständen der großen deutschen Industrieunternehmen durchgesetzt. Dabei ist das Leitbild des Shareholder Value in den unternehmensinternen Entscheidungsprozessen nicht zu der einzigen relevanten Begründungsordnung geworden. Daneben sind nach wie vor z. B. Überlegungen der technischen Exzellenz, Markterwartungen oder auch nur das Bauchgefühl der entscheidenden Führungskräfte relevant (vgl. Kädtler 2009). Allerdings haben die dem Shareholder-Value-Leitbild entsprechenden Rationalitäts- und Legitimitätskriterien gegen Ende der 1990er Jahre und zu Anfang des neuen Jahrzehnts auch in deutschen börsennotierten Konzernen stark an Bedeutung gewonnen. Eine wichtige Triebfeder dieses Wandels, der als zumindest partielle Durchsetzung des Finanzmarktkapitalismus beschrieben wird (vgl. u. a. Windolf 2005), war zweifellos die schnelle Verbreitung großzügiger Aktienoptionspläne nach US-amerikanischem Vorbild. Wenn Vorstände derart stark von Aktienkurssteigerungen profitieren können, was sollte sie dann noch davon abhalten, das 
entsprechende Leitbild unternehmerischen Handels zu übernehmen oder zumindest doch zu versuchen, eine solche Übernahme des Leitbilds den Anlegern glaubwürdig $\mathrm{zu}$,kommunizieren'?

Eine weitere finanzwirtschaftliche Entwicklung, welche in Zukunft die Langfristorientierung großer deutscher Kapitalgesellschaften erschweren könnte, ist der Anstieg ihres Verschuldungsgrades. ${ }^{15}$ Traditionell gelten deutsche Unternehmen im internationalen Vergleich als relativ stark verschuldet. Zurückgeführt wird dies vor allem auf die Hausbankbeziehungen, die den deutschen Unternehmen eine verlässliche Quelle externer Finanzierung bieten. Mit der Stärkung der Wertpapiermärkte sind für Großunternehmen zusätzliche Möglichkeiten der externen Finanzierung entstanden. Zugleich werden ihnen auch neue Möglichkeiten eröffnet, Ausgaben zu tätigen. Das gilt in besonderem Maße für den Aktienmarkt. In dem neuen finanzwirtschaftlichen Kontext entwickelter Wertpapiermärkte gibt der gesamte Sektor der nicht-finanziellen Kapitalgesellschaften in fast jedem Jahr mehr Geld für die Pflege des eigenen Aktienkurses durch Rückkaufprogramme und für die Übernahme anderer Unternehmen aus, als er durch die Ausgabe neuer Aktien einnimmt. ${ }^{16}$ Aus diesem Grund führt der Bedeutungszuwachs des Aktienmarktes in einer Volkswirtschaft keineswegs zu einer Stärkung der Eigenkapitalbasis börsennotierter Großunternehmen, sondern paradoxerweise zu einem Anstieg ihres Verschuldungsgrades. Verstärkt wird dieser Trend dadurch, dass sich mit dem Bedeutungszuwachs der Aktienmärkte auch noch zwei andere kostspielige Praktiken immer weiter ausbreiten: die bereits erwähnten Aktienoptionsprogramme für Manager und die Zahlung hoher Dividenden, mit denen auch in Phasen rückgängiger Gewinne der Aktienkurs stabilisiert werden soll.

Im Shareholder-Value-Leitbild unternehmerischen Handelns wird der Anstieg des Verschuldungsgrades, wie er sich bei den großen deutschen Kapitalgesellschaften zeigt, als positiv eingeschätzt. Gemäß der sogenannten ,free cash flow-Hypothese'

15 Für die Jahre seit 1997 veröffentlicht die Deutsche Bundesbank (2009b) mittlerweile auch nach Größe und Rechtsform getrennte Durchschnittswerte wichtiger Unternehmenskennzahlen. Diese lassen für große Kapitalgesellschaften in Deutschland zwischen 1997 und 2007 einen Anstieg des Verschuldungsgrads (Anteil der kurz- und langfristigen Schulden an der Bilanzsumme) von 42,9\% auf 45,8\% erkennen (ebd. 73). Dieser Anstieg ist auch deshalb markant, weil er gegenläufig zum Trend eines sinkenden Verschuldungsgrads für den Unternehmenssektor in Deutschland insgesamt (von 64,5\% auf 56,2\%) ist (ebd. 21). Mit Blick auf den hier interessierenden finanzwirtschaftlichen Kontext der geschäftspolitischen Strategien deutscher Unternehmen wäre es natürlich interessant zu untersuchen, ob bei dem Rückgang des Verschuldungsgrads des Unternehmenssektors insgesamt (ggf. neben einer verbesserten Ertragslage auch) ein schlechterer Kreditzugang von KMU durch Rückgang bzw. Beeinträchtigungen von Hausbankbeziehungen eine Rolle spielt.

16 Für deutsche nichtfinanzielle Kapitalgesellschaften in den Jahren 1991 bis 2008 zeigt das die Gesamtwirtschaftliche Finanzierungsrechnung (Deutsche Bundesbank 2009a: 22, 40-52). In dem hier erfassten Zeitraum nahmen diese Unternehmen nur in vier Jahren mehr durch Aktienemissionen ein als sie bei Aktienkäufen (nach Abzug der Einnahmen aus Aktienverkäufen) ausgaben. Der jährliche Abfluss von Finanzmitteln aus diesem Unternehmenssektor auf die Aktienmärkte lag in den untersuchten 18 Jahren bei durchschnittlich knapp 24 Mrd. $€$ (bei steigender Tendenz und mit besonders starken Abflüssen in Zeiten der Aktienhausse). Noch ausgeprägter ist der negative Einfluss des Aktienmarktes auf die Eigenkapitalbasis beim Nonfarm-NonfinancialCorporate-Business in den USA (Federal Reserve Board 2009, Tafel F. 102). 
sinke so jener Teil der Einnahmen, über den das Management frei und von Kapitalgebern unkontrolliert verfügen könne (vgl. Jensen 1986). Allerdings geht mit der höheren Verschuldung bei der gleichen Entwicklung der Einnahmen auch ein höheres Konkursrisiko einher. Dies wirkt sich negativ auf die Bereitschaft der Manager aus, Geschäftsstrategien zu wählen, bei denen sich Investitionen erst nach einer langen Anlaufphase auszahlen werden. Schließlich werden aus Sicht der - am Erhalt ihrer Positionen interessierten - Vorstände mit der steigenden Verschuldung langfristig angelegte Geschäftsstrategien riskanter.

Untersuchungen in den USA zeigen, dass viele Manager großer Aktiengesellschaften, die regelmäßig Quartalsberichte vorlegen, ihre unternehmerischen Entscheidungen stark an den voraussichtlichen Folgen für den Gewinn in der laufenden Periode (,earnings per share') ausrichten. Um einen Absturz des Aktienkurses zu verhindern, wird beinahe alles dafür getan, dass das Ergebnis des entsprechenden VorjahresQuartals, vor allem aber die vorherrschende Gewinnprognose der Analysten nicht unterschritten wird. Notfalls unterbleiben sogar Investitionen (z.B. in Forschung und Entwicklung), die den künftigen Unternehmenswert erheblich steigern könnten (vgl. Graham et al. 2005; Cheng et al. 2005). Obwohl der US-Aktienmarkt kaum noch als Markt für Unternehmenskontrolle fungiert, ist die Orientierung der Vorstände am Aktienkurs und die mit ihr einhergehende Vernachlässigung langfristiger Unternehmensziele unter großen US-amerikanischen Unternehmen sehr verbreitet.

So weit ist die Erosion der Langfristorientierung in deutschen Unternehmen sicher nicht fortgeschritten! Konsequent am Shareholder-Value-Leitbild, welches das Verfolgen von Geschäftsstrategien erschwert, die sich erst nach einer längeren Verlustphase auszahlen, haben sich in Deutschland wohl nur wenige Unternehmen orientiert. Die drei oben erwähnten Gruppen von Unternehmen - die börsennotierten AGs, deren Aktien durchgängig in Streubesitz sind, sowie die KMU und die abgespaltenen Konzernteile, die in der Hand von Finanzinvestoren sind oder deren Eigentümer vergleichsweise schnell wechseln - sind insgesamt vergleichsweise klein. Die meisten deutschen Unternehmen haben die Shareholder-Value-Strategien gar nicht oder allenfalls abgewandelt und abgemildert übernommen (vgl. Faust/Kädtler 2009). Die Zeit, in der in Deutschland wirtschaftsliberale Plausibilitäten einschließlich des Unternehmensleitbilds eindeutig im Vormarsch waren, blieb insgesamt auf wenige Jahre rund um die Jahrtausendwende begrenzt. Schon bald trübten die Börsentalfahrt, die SchlieBungen größerer Werke wegen Produktionsverlagerung ins Ausland sowie die Diskussionen über hohe Managergehälter die Aussichten der marktradikalen Wirtschaftsliberalen die Mehrheit der Bevölkerung für ihre Projekte zu gewinnen, auch wenn dies von den führenden Parteien erst mit erheblicher Verspätung registriert wurde (vgl. ebd.).

Die globale Finanzkrise hat den Bedeutungsgewinn der kapitalmarktbezogenen Segmente der Finanzwirtschaft, der sich in den letzten Jahren deutlich abzeichnete, erst einmal unterbrochen. Das erschwert Prognosen über die weitere Entwicklung der Finanzwirtschaft in Deutschland, in Europa und weltweit - und damit auch über die künftigen Veränderungen des finanzwirtschaftlichen Kontextes der Renditeorientierung deutscher Großunternehmen. Folgt man der Wirtschaftspresse, dann ist mit Blick auf die Bedeutung des Shareholder-Value-Leitbilds das Bild deutscher Aktienge- 
sellschaften in der Wirtschaftskrise durchaus zwiespältig zu nennen. Einerseits nutzten die Manager vieler Konzerne extensiv das von der Regierung mehrfach verlängerte Angebot des Kurzarbeitergelds und signalisierten damit, dass sie die essentielle Bedeutung der Mitarbeit erfahrener und qualifizierter Arbeitnehmer für den künftigen wirtschaftlichen Erfolg ihres Unternehmens erkannt hatten. Andererseits beschlossen nicht wenige Unternehmensleitungen hohe Ausschüttungen und zeigten sich insofern entschlossen, die Aktionäre notfalls auch mit Maßnahmen bei Laune zu halten, die an die Substanz des Unternehmens gehen, nämlich in ertragsschwachen Zeiten die Möglichkeiten, rentable Investitionen zu tätigen, einschränken und so die längerfristigen Gewinnaussichten trüben.

\section{3 Übersteigerter Renditedruck}

Wie im vorhergegangenen Abschnitt dargestellt, hat sich in der Bundesrepublik in den zehn Jahren vor der Krise das Shareholder-Value-Leitbild unternehmerischen Handelns nicht nur über die Medien, auf Tagungen, durch Unternehmensberatungen und in den internen Beratungsprozessen der Vorstände und Aufsichtsräte ausgebreitet. Vielmehr wurde es auch im Unternehmenssektor institutionell verankert. Neben der Ausbreitung von Aktienoptionsplänen sind hier die Überführung einiger börsennotierter AGs in vollständigen Streubesitz und die Entstehung eines Marktes zu nennen, auf dem KMU oder einzelne Unternehmensteile von Finanzinvestoren oder Konzernen als Vermögensobjekte gekauft und bald wieder verkauft werden. Dabei drängte das Leitbild die Manager zu einer Geschäftspolitik, die tendenziell nicht nur kurzatmig ist, sondern auch einseitig sehr bohe Gewinnziele verfolgt. Abgesehen von kosmetischen bzw. kommunikationsstrategischen Veränderungen, die isoliert auf die Höhe des Aktienkurses selbst zielen, bestehen die Shareholder-Value-Strategien vor allem darin, dem Unternehmen insgesamt, aber auch allen Töchtern und Abteilungen eine sehr hohe Eigenkapitalrendite als Ziel vorzugeben, das unter fast allen Umständen erreicht werden muss. Damit hat sich ausgerechnet der Renditeanspruch der Eigenkapitalgeber, der in der Betriebswirtschaftslehre eigentlich als Residualgröße begriffen wird, zu einer verbindlichen Vorgabe entwickelt, während alle Kostengrößen - allen voran die Arbeitskosten - als variabel begriffen werden (vgl. Kädtler 2009). Vor allem nach dem Zusammenbruch der neuen kapitalmarktdominierten Finanzwirtschaft wundert man sich über die Höhe dieser Gewinnvorgaben. Kontinuierlich sollten Renditen in Höhe von 10\%, 15\% oder mehr Prozent erwirtschaftet werden. In den Jahren vor der Krise mag das durch Beteiligung an mehr oder minder spekulativen Finanzgeschäften dem einen oder anderen Großunternehmen als Ganzem gelungen sein. Aber auf die Dauer ist es ohne eine massive Umverteilung zugunsten der Vermögensbesitzer rechnerisch unmöglich, dass alle (oder die meisten) Investoren Renditen erzielen, die deutlich oberhalb der Wachstumsrate des Weltsozialprodukts liegen (Thielemann/Ulrich 2003: 59). Wie konnte es dazu kommen, dass mittel- und langfristig derart überfordernde Renditevorgaben weithin als realistische Unternehmensziele wahrgenommen wurden?

Neben der Ausbreitung des Shareholder-Value-Leitbilds gibt es mehrere wirtschaftliche Entwicklungstrends, mit denen man die hohen Gewinnziele der letzten Jahre möglicherweise erklären kann. Zu den realwirtschaftlichen Veränderungen, die plausible Erklärungsansätze bieten, gehören die Produktivitätssteigerungen durch die Aus- 
breitung der neuen Informationstechnologie, aber auch die Schwächung der Gewerkschaften, die man u. a. auf die hohe Massenarbeitslosigkeit, die gestiegene Konkurrenz auf den internationalen Gütermärkten und die Dominanz wirtschaftsliberaler Positionen in der politischen Öffentlichkeit zurückführen kann. Von erheblicher Bedeutung dürfte es aber auch sein, dass die meisten Akteure in der Finanzbranche tatsächlich bis zum Ausbruch der globalen Finanzkrise exorbitant hohe Gewinne erzielen konnten. So sind in den USA zwischen 1980 und 2005 die Gewinne in der Finanzwirtschaft etwa dreimal so schnell gestiegen wie das nominale Bruttoinlandsprodukt des Landes. ${ }^{17}$ Weil auf den Kapitalmärkten die Kapitalgesellschaften verschiedener Branchen über Aktien und Rentenpapiere direkt miteinander konkurrieren, geht von den übersteigerten Eigenkapitalrenditen in der Finanzwirtschaft eine Sogwirkung aus, welche die Renditeerwartungen an alle Kapitalgesellschaften in die Höhe zieht. Dieser Entwicklungstrend, der einen finanzwirtschaftlichen Erklärungsbeitrag für die Höhe der Renditeanforderungen darstellt, soll im Folgenden noch etwas deutlicher herausgearbeitet werden.

Die extrem hohen Gewinne, die in den Industrieländen viele größere Finanzinstitute bis 2006 erzielen konnten, müssen heute als Teilaspekt einer tiefgreifenden Fehlentwicklung der Finanzwirtschaft begriffen werden, die in der globalen Finanzkrise offen zu Tage getreten ist. Sie gehen nämlich darauf zurück, dass seit etwa zwanzig Jahren eine große Preisblase bei international gehandelten Vermögensgütern entstanden, immer weiter gewachsen und dabei ,über' verschiedene Märkte (Aktienmärkte, Immobilienmärkte einiger Länder und Rohstoffterminmärkte), gewandert' ist, aus der in der Krise allmählich die Luft entweicht.

In diesem Beitrag kann das vielfältige Ursachengeflecht für die globale Finanzkrise nicht dargestellt werden. ${ }^{18}$ Immerhin sei erwähnt, dass bei der Entstehung und dem Wachstum der großen Preisblase auf den Vermögensmärkten die neue Gesamtkonstellation der Finanzwirtschaft eine zentrale Rolle spielt. Diese zeichnet sich, wie oben skizziert (3.1), durch ein dynamisches Investmentbanking aus, das einen Großteil der Vermögenspositionen ständig umschichtet und mit dem Sektor der Geschäftsbanken eng verbunden ist. Verständlich wird die Bedeutung dieser Konstellation für die Renditehöhe, wenn man die Fähigkeit der Geschäftsbanken, selbständig Geld zu schaffen, als Ausgangspunkt nimmt: Geld kommt vor allem dadurch in die Wirtschaft, dass die Geschäftsbanken Kredite vergeben. Bei einer Kreditvergabe räumt die Bank dem Kreditkunden auf dem Girokonto ein Guthaben ein, über das er verfügen kann. Da Giroeinlagen heute das wichtigste Zahlungsmittel und mithin selbst Geld sind, entsteht bei der Kreditvergabe also neues Geld. Das ist auch dann der Fall, wenn die Kreditinstitute von anderen wirtschaftlichen Akteuren Vermögensgüter (z. B. Aktien oder Immobilien) kaufen. In diesem Fall ist es der Verkäufer des Vermögensgutes, der

17 Vgl. Tafel F.7 der US-amerikanischen Flow of Funds Accounts (Federal Reserve Board 2009).

18 Aus der Flut der einschlägigen Veröffentlichungen seien genannt: Burghof/Prothmann 2008; Sinn 2009; Nastansky u. a. 2009 und vor allem BIZ 2009. Für ein Verständnis der Logik von Finanzkrisen sind m. E. die Arbeiten von Hyman P. Minsky (vgl. Minsky 1982; 1986; Emunds 2000: 179-230) bedeutsam. Vgl. auch meine an Minsky anschließenden Bemühungen um eine Diagnose, die bei den Ursachen der Finanzkrise das heute oft nicht beachtete Geldschöpfungspotenzial der Geschäftsbanken ansetzt: Emunds 2008; 2009a; Emunds/Reichert 2009. 
auf seinem Girokonto eine Guthabenbuchung erhält. Ohne die Geldschöpfung der Geschäftsbanken käme in einer hoch-arbeitsteiligen Wirtschaft das wirtschaftliche Geschehen gar nicht erst in Gang. Zugleich ist die Fähigkeit der Banken, neues Geld zu schaffen und in Umlauf zu bringen, aber auch ein Einfallstor für grundlegende wirtschaftliche Fehlentwicklungen. Die Geschäftsbanken können den anderen wirtschaftlichen Akteuren nämlich auch mehr Geld zur Verfügung stellen, als diese benötigen, um die bereitgestellten (bzw. aktuell überhaupt bereitstellbaren) Güter zu kaufen. Wird dieses überschüssige Geld für Käufe auf den Gütermärkten verwendet, dann kommt es zu einer Übersteigerung der Konjunktur und Inflation: Das Niveau der Preise für die Güter der ,laufenden Produktion' steigt. Werden mit ihm jedoch Vermögenswerte wie Aktien, Immobilien oder Rohstoff-Terminkontrakte gekauft, dann wächst auf diesen Märkten eine Preisblase.

Ausdehnung der Geldmenge und Entstehung von Preisblasen auf Vermögensmärkten mit anschließendem ,Crash' - ein solches Szenario ist für den Weg in eine Finanzkrise und deren Ausbruch typisch (vgl. Minsky 1982; Kindleberger 1996). Solche Finanzkrisen hat es in kleinerem Maßstab vor den 1930er und - vor allem in peripheren Ländern - seit den 1990er Jahren immer wieder einmal gegeben. Sieht man von dem Börsenkrach $1929 \mathrm{ab}$, dann erreichte aber der weltweite Zusammenbruch der Vermögenswerte in der offenbar nun ausklingenden Finanzkrise eine Dimension, die weit über die Verluste in den anderen Krisen hinausgeht. Dass sich in den 1980er und 1990er Jahren die Konstellation der neuen kapitalmarktdominierten Finanzwirtschaft durchsetzte, also ein sehr dynamisches Segment des Investmentbanking entstand, das mit den kreditvergebenden Geschäftsbanken eng verflochten ist, bietet eine plausible Erklärung für die Größe dieser Preisblase - und damit auch für die erstaunliche Höhe der Gewinne in der Finanzwirtschaft in den Jahren vor der Krise.

Über Kredite an die Käufer von Vermögenswerten und dadurch, dass sich die Geschäftsbanken selbst an den Wertpapier- und Immobiliengeschäften beteiligten, sind nämlich immer mehr Geldmittel auf diese Märkte geflossen und haben dort für einen langfristigen Anstieg der Vermögenspreise gesorgt. Schaut man auf die Entwicklung der Aktienkurse und Immobilienpreise in großen Industrieländern sowie auf die Kursentwicklung auf einigen Rohstoff-Terminmärkten, dann zeigt sich, dass dieser Anstieg zwar auf den Aktienmärkten von Phasen vorübergehender Kursverluste unterbrochen wurde, aber insgesamt bis Mitte $2007 \mathrm{zu}$ sehr hohen Vermögenswerten führte (vgl. Borio/Drehmann 2009: 15; Belke/Gros 2010). Mit diesem Wachstum der Preisblase ging eine bezeichnende Entwicklung bei den Krediten der Geschäftsbanken in den Industrieländern einher: Deren Volumen wuchs sehr viel schneller als das Bruttoinlandsprodukt der betreffenden Länder.

Das Wachstum der globalen Preisblase in den letzten 20 Jahren ist so umfassend, dass es kaum auf kreditfinanzierte spekulative Transaktionen allein zurückgeführt werden kann. Schließlich sollte man als ,Spekulation' ausschließlich den gezielte Versuch eines Marktteilnehmers bezeichnen, durch geschicktes Ausnutzen kur:fristiger Preisschwankungen Gewinne zu erzielen. ${ }^{19}$ Neben spekulativen Marktaktivitäten ist vielmehr auch

19 Vgl. Chick 1990 und Emunds 2000: 167-172. Wenn man mit dem Begriff ,Spekulation` spezifische wirtschaftliche Handlungen bezeichnen will, sollte man ihn vergleichsweise eng definieren. 
zu berücksichtigen, dass es zu den Kernaktivitäten der kapitalmarktbezogenen Segmente der Finanzwirtschaft gehört, Vermögenspositionen ständig neu zu arrangieren. Die Portefeuilles der Fonds und vieler vermögender Haushalte werden durch Umschichtungen kontinuierlich ,optimiert'; und die Konzerne werden von den Investmentbankern so beraten, dass sie immer wieder Unternehmensteile abstoßen und neue hinzukaufen oder mit anderen Konzernen fusionieren. Bei diesen Umschichtungen von Vermögenswerten wurde zumeist auch deren Finanzierung ,optimiert' und dabei der Anteil, den die Käufer nicht aus Eigenmitteln, sondern durch Aufnahme neuer Schulden finanzierten, immer weiter erhöht. Durch die Verbindung zwischen dem Segment der Geschäftsbanken und dem der Investmentbanken waren die zusätzlichen Kreditmittel leicht aufzutreiben. Das bei diesen Aktivitäten neu geschaffene Geld ermöglichte dann den Anstieg der Vermögenspreise sowie die extrem hohen Gewinne im Finanzsektor und die Spitzeneinkommen vieler Banker.

\section{Konsequenzen für die Wirtschafts- und Unternehmensethik}

Die Wirtschafts- und Unternehmensethik zielt darauf ab, dasjenige wirtschaftliche Handeln zu fördern, das die Überlebenschancen sowie die Entfaltungs- und Beteiligungsmöglichkeiten von Personen mehrt $^{20}$ und dabei die vitalen Lebensinteressen aller heute oder künftig lebenden Menschen nicht beeinträchtigt. Wählt man einen sehr weiten Vermögensbegriff, der alle künftigen Lebenschancen von Menschen umfasst, dann kann man wichtige Teilaspekte der damit umrissenen Zielvorstellung auch als umfassenden Aufbau von Vermögen beschreiben. Die einzelnen wirtschaftlich Handelnden, allen voran diejenigen, die die Geschäftsstrategien von Unternehmen gestalten, sollen in diesem Sinne zu einem Aufbau von Vermögen beitragen und sind darüber hinaus angehalten, alle Handlungsweisen bzw. Geschäftsstrategien zu meiden, die einem solchen Aufbau langfristig zuwiderlaufen. Sie sind dazu angehalten, weil die Anerkennung der vitalen Lebensinteressen aller Menschen sie ethisch verpflichtet und nicht weil sich dies für sie bzw. für ihr Unternehmen auf die Dauer auszahlt.

Mit der Leitung eines Unternehmens ist jedoch die Aufgabe verbunden, dafür zu sorgen, dass sich das Unternehmen dauerhaft am Markt behaupten kann. Deshalb tun diejenigen, die ethisch sinnvolles Wirtschaften fördern wollen, gut daran, den Unternehmensleitungen auch nahe zu bringen, dass entsprechende Geschäftsstrategien wirtschaftlich erfolgversprechend sein können. Da die Kosten solcher Strategien sofort anfallen, die positiven Wirkungen auf die Motivation der Mitarbeiter bzw. auf die Reputation des Unternehmens und damit ggf. auf die Geschäftsentwicklung nur für die Zukunft erhofft werden können, geht es dabei um den widersprüchlichen $\mathrm{Zu}$ sammenhang zwischen der betriebswirtschaftlichen Logik in der kurzen (Kostenvermeidung) und in der langen Frist (Ausgaben zum Erschließen künftiger Möglichkeiten der Wertschöpfung bzw. des Gewinns). Insofern liegt es nahe, in einem ökonomi-

Verwendet man ,Spekulation' dagegen für jeden Versuch, Preisunterschiede auszunutzen, dann ist beinahe alles Wirtschaften Spekulation oder enthält zumindest ein spekulatives Moment. keiten von Menschen zuerst durch das Angebot benötigter oder gewünschter Güter und durch gerechte Arbeitsbedingungen. 
schen Sprachspiel die Wahl ethisch gebotener oder wünschenswerter Geschäftsstrategien als eine Investition zu interpretieren.

Geschwächt wird diese Argumentationsstrategie natürlich dadurch, dass (fast immer) offen bleiben muss, ob sich die jeweilige Investition tatsächlich für das Unternehmen ,lohnt', also ob den Ausgaben in Gegenwart (und Zukunft) tatsächlich größere Einnahmesteigerungen in der Zukunft gegenüberstehen werden. Das liegt nicht nur daran, dass es schwer fällt, letztere zu quantifizieren. Vielmehr ist auch der Unterschied zwischen der gesellschaftlichen bzw. gesamtwirtschaftlichen und der betriebswirtschaftlichen Ebene zu beachten. Dass der Raubbau am Natur- oder Arbeitsvermögen langfristig negativ ,zurückschlägt', während Ausgaben für deren Erhalt bzw. Aufbau insgesamt der Vermögensakkumulation im umfassenden Sinne dienlich sind, dürfte für die Gesellschaft insgesamt und damit wohl auch für den Unternehmenssektor als ganzen offensichtlich sein. Offen dagegen ist die Frage, ob die negativen Rückkopplungseffekte von Raubbau-Strategien und die positiven Wirkungen langfristorientierter investiver Geschäftsstrategien wirklich bei den Unternehmen selbst, welche diese oder jene Strategien einschlagen, so stark zu Buche schlagen, dass die höheren Ausgaben für sozial und ökologisch verantwortliches Wirtschaften auf die Dauer durch höhere Einnahmen überkompensiert werden.

Herauszustellen, dass ethisch vorzugswürdige Geschäftsstrategien langfristig auch wirtschaftlich besonders erfolgreich seien, ist insofern eine zwar sinnvolle, aber eben auch mit erheblichen Problemen belastete Argumentationsstrategie der Unternehmensethik. ${ }^{21}$ Darüber hinaus wird sie - das war das zentrale Thema dieses Beitrags in der Gegenwart weiter geschwächt. Die Ausbildung einer neuen kapitalmarktdominierten Variante der Finanzwirtschaft und, eng damit verbunden, die Ausbreitung des Shareholder-Value-Leitbilds unternehmerischen Handelns haben in den Jahren vor Ausbruch der Finanzkrise forciert, dass sich auch in Deutschland viele Manager groBer Aktiengesellschaften vorrangig oder fast ausschließlich an einem Ziel orientieren: jeweils im laufenden Quartal und in nächster Zukunft für die Aktionäre extrem hohe Renditen zu erzielen. Die Höhe dieser Renditeziele ließ kaum Spielraum für konsensbzw. kompromissorientierte Arrangements mit den Arbeitnehmern und anderen Stakeholdern, die nicht Shareholder sind. Die kurze Frist, die man sich unter dem realen oder auch nur vermeintlichen Druck der großen institutionellen Anleger zum Erreichen der ehrgeizigen Renditeziele setzte, sorgte dafür, dass langfristige Erwägungen zum Aufbau guter Ertragsmöglichkeiten in der ferneren Zukunft kaum mehr in die betriebswirtschaftlichen Entscheidungsprozesse eingingen. Zu letzterem zählen eben auch Überlegungen, durch ethisch vorzugswürdige Geschäftsstrategien die Motivation von Mitarbeitern oder die Reputation des Unternehmens überhaupt zu steigern.

Angesichts des Strukturbruchs, den die globale Finanzkrise möglicherweise bedeutet, muss es offen bleiben, ob sich in den nächsten Jahren die hier skizzierten Trends, welche die Finanzwirtschaft vor Ausbruch der Krise kennzeichneten, fortsetzen wer-

21

Ein weiteres, hier nicht behandeltes, Problem dieser Argumentationsstrategie besteht darin, dass dies nur für die Berücksichtigung der Interessen solcher Akteure (,stakeholder) gilt, die die Macht haben (z. B. über Kaufkraft verfügen) bzw. in Zukunft haben können, den künftigen Unternehmenserfolg zu beeinflussen (vgl. Thielemann/Breuer 2000). 
den. Wie werden sich künftig in der Finanzwirtschaft die Gewichte zwischen dem kapitalmarktbezogenen Segment und dem Geschäftsbankensegment verteilen? Welche Entwicklungen werden diese Teilbereiche der Finanzwirtschaft durchlaufen? Wird der internationale Trend, dass sich beide miteinander verflechten, weiter fortgesetzt? Werden die Finanzinstitute auch in Zukunft wieder stark auf Geschäftsstrategien setzen, bei denen ein Großteil der Gewinne aus Preissteigerungen auf den Vermögensmärkten resultiert, die sie durch eine Ausdehnung ihrer Geldschöpfung selbst hervorgerufen haben? Wie werden sich diese Entwicklungen des finanzwirtschaftlichen Kontextes auf den Zeithorizont der Geschäftsstrategien deutscher Großunternehmen auswirken? Die Antworten auf diese Fragen hängen vor allem von den gegenwärtigen politischen Entscheidungen über die Regulierung der Finanzwirtschaft ab - ein wichtiges Themenfeld für die wirtschaftsethische Orientierung, das in diesem Beitrag nicht bearbeitet werden kann. ${ }^{22}$ Hier ging es nur darum, dass diese Trends dann, wenn sie sich fortsetzen, die Unternehmensethik, insbesondere die Argumentationsstrategie der langfristig lohnenswerten Investitionen, in Ethik' erschweren werden.

Wenn hier behauptet wird, dass heute vom finanzwirtschaftlichen Kontext unternehmerischer Strategien her die Chancen für erfolgreiche unternehmensethische Beratung geringer sind als früher, soll damit natürlich nicht ausgesagt werden, dass diese nicht notwendig sei. Mit Gesetzen allein lässt sich wirtschaftliches Handeln nicht in sozialund umweltverträgliche Bahnen lenken. Das zeigen auch jene Entwicklungen der Finanzwirtschaft, die in die globale Finanzkrise führten. Zwar geht es bei der starken Expansion der Finanzwirtschaft und der rapiden Akkumulation von Risiken in den Finanzinstituten zuerst darum, dass die gesetzlichen Regeln unzureichend waren und nicht konsequent durchgesetzt wurden. Zugleich aber waren diese Entwicklungen auch möglich, weil die Finanzinstitute, schnelle und hohe Gewinne vor Augen, die bestehenden gesetzlichen Regelungen zur Risikobegrenzung systematisch umgingen. Die Regeln wurden also nur dem Buchstaben, nicht auch dem Geiste nach eingehalten. Damit sie auch dem Geiste nach beachtet werden, bedarf es in den Finanzinstituten einer anderen Unternehmenskultur - eben einer Unternehmenskultur des langfristigen wirtschaftlichen Erfolgs. Schließlich werden die Vorstände (und Mitarbeiter) der Institute dann, wenn sie eine Langfristperspektive einnehmen, auch wahrnehmen, dass die Einhaltung der risikobegrenzenden Regulierung in ihrem eigenen Interesse liegt. Allerdings muss die Ausbreitung einer solchen Unternehmenskultur durch entsprechende Regeln gefördert werden. Intern geht es dabei um die derzeit viel diskutierten Strukturen der Boni und Provisionen, extern u. a. um die hier behandelten Renditeanforderungen der Eigenkapitalgeber, die eben auch in den Finanzinstituten die Wahl bestimmter Geschäftsstrategien nahelegen und andere weniger attraktiv erscheinen lassen. Der innere Zusammenhang zwischen geeigneten gesetzlichen Regeln und verantwortlich handelnden Akteuren lässt sich also nicht einseitig auflösen - auch nicht zugunsten der Regeln! 23

Dass dieser Zusammenhang nicht einseitig zugunsten gewissenhafter (oder auch nur besonders gut, weil besonders lange kalkulierender) Akteure in den Vorstandsetagen

22 Vgl. die entsprechenden Hinweise in Emunds 2009a; 2009b.

23 Für die Finanzwirtschaft vgl. u. a. Koslowski 1997, sowie Reichert 2009. 
der Unternehmen aufgelöst werden soll, dürfte in der deutschsprachigen Wirtschaftsund Unternehmensethik kaum umstritten sein. Wohl aber ist in der neueren Literatur die Auffassung verbreitet, dass in den Zeiten der Globalisierung der unternehmensethische Weg der ethischen Beratung von ,Führungskräften' an Bedeutung gewonnen, der wirtschafts- bzw. institutionenethische Weg, bei dem es vor allem um Beiträge zur politisch-öffentlichen Debatte über die richtigen Regeln geht, an Bedeutung verloren habe. Der Trend, stärker auf Unternehmensethik zu setzen, wurde in diesem Beitrag kritisiert. Nicht nur mit der Skizze einer grundsätzlichen Reflexion auf die Schwierigkeiten, die in kapitalistischen Marktwirtschaften immer mit dem langfristigen Aufbau von Vermögen im umfassenden Sinne verbunden sind, sondern auch mit Überlegungen zur gegenwärtigen Entwicklung des Unternehmenssektors. Bei letzterem stand im Mittelpunkt, dass sich in den Jahren vor dem Ausbruch der globalen Finanzkrise der finanzwirtschaftliche Kontext für die Geschäftsstrategien von Großunternehmen grundlegend verändert hat. Die ,Zeiten der Globalisierung' sind eben auch die Zeiten einer neuen Form der Finanzwirtschaft, die es den Unternehmensethikerinnen und -ethikern nicht leichter macht!

Aus Sicht der Politischen Wirtschaftsethik, die eingangs skizziert wurde, ist aber in vielen neueren einschlägigen Veröffentlichungen nicht nur das Vertrauen in die Möglichkeiten der Unternehmensethik zu hoch, sondern auch die Erwartung an die gegenwärtigen Chancen für eine Wirtschaftsethik als Institutionenethik zu gering. Natürlich werden die nationalstaatlichen Regierungen durch die Internationalisierung der Wirtschaft in ihren Möglichkeiten, das Handeln der einzelwirtschaftlichen Akteure auf das Gemeinwohl hin zu lenken, geschwächt. Keine von ihnen ist heute noch in der Lage, ,ihre' Volkswirtschaft im Alleingang souverän zu gestalten. Aber entsteht bei den Grundlagenreflexionen der Unternehmensethik nicht manches Mal ein zu negatives Bild von den Möglichkeiten der Einflussnahme, welche die Regierungen der Nationalstaaten auch heute noch haben? Die politischen Administrationen der großen Industrie- und Schwellenländer können, wenn sie sich untereinander absprechen und die, von ihnen weithin beherrschten, multilateralen Organisationen einbeziehen, nach wie vor eine hohe Lenkungswirkung erzielen. Außerdem ist die Internationalisierung der Wirtschaft nur zum kleineren Teil in dem Sinne eine Globalisierung, dass die Interaktionen und Handlungsketten nicht nur die Grenzen eines Staates, sondern auch die einer Großregion überschreiten. Gerade bei den wirtschaftlichen Akteuren in Europa nehmen vor allem die grenzüberschreitenden Transaktionen mit Vertragspartnern aus anderen EU-Mitgliedsländern zu. Insofern steigt die wirtschaftliche Integration besonders stark auf der Ebene der Großregion, auf der es bereits politische Institutionen gibt, die prinzipiell zu einer effektiven Lenkung des Wirtschaftens in der Lage wären. Das Problem scheint weniger grundsätzlich in einer massiv verringerten Steuerungskapazität (supra-)staatlicher Institutionen zu liegen, als darin, dass unter dem Einfluss wirtschaftsliberaler Positionen der Wille der politischen Eliten wenig ausgeprägt ist, die Geschäftsstrategien der Konzerne durch gesetzliche Regeln effektiv zu lenken. Aber selbst dann, wenn man nicht nach den prinzipiellen Möglichkeiten der Steuerung, sondern nach der faktischen Entwicklung fragt, wird man feststellen müssen, dass der befürchtete große ,race to the bottom' bei den Sozial- und Umweltregeln bisher ausgeblieben ist. 
Das bedeutet keineswegs, dass bei der internationalen politischen Steuerung des Wirtschaftens alles zum Besten bestellt wäre. Im Gegenteil: So gibt es z. B. nach wie vor in vielen Ländern keine Sozialstandards, welche die Erwerbstätigen effektiv vor Ausbeutung schützen (vgl. Sachverständigengruppe 2008). Und die Regierungen der großen Industrieländer haben sich bisher nicht zu Maßnahmen verpflichtet, die zu einer massiven Reduktion der Emissionen klimaschädlicher Gase führen würden, ohne die eine Erwärmung des Weltklimas um deutlich mehr als zwei Grad Celsius nicht verhindert werden kann (vgl. u. a. Leggewie/Welzer 2009). Aber diese und andere massive Fehlentwicklungen verweisen vor allem auf (bisher) verpasste Möglichkeiten der politischen Steuerung und nicht auf den vollständigen Verlust oder die massive Einschränkung der Steuerungskapazität (supra-)staatlicher politischer Institutionen. Vor allem wird nicht plausibel, warum Verbesserungen zuerst von unternehmensethischen Versuchen erwartet werden, die Manager der großen Konzerne von ihrer hohen sozialen und ökologischen Verantwortung zu überzeugen, und warum es heute weniger aussichtsreich sein soll als früher, mit - theoretisch überzeugenden - wirtschaftsethischen Argumenten dazu beizutragen, dass der politische Druck auf demokratische Regierungen steigt, die für eine sozial und ökologisch verträgliche Ausrichtung des Wirtschaftens dringend benötigten Maßnahmen auch wirklich zu beschließen.

\section{Literaturverzeichnis}

Adams, M. (1994): Die Usurpation von Aktionärsbefugnissen mittels Ringverflechtung in der „Deutschland AG“. Vorschläge für Reformen im Wettbewerbs-, Steuer- und Unternehmensrecht, in: Die Aktiengesellschaft, Jg. 39/Heft 4, 148-158.

Allen, F./ Douglas, G. (2000): Comparing Financial Systems, Cambridge/Mass.: MIT-Press.

Aßländer, M. S./ Brink, A. (2008): Begründung korporativer Verantwortung: Normenkonkretion als Prozess, in: Scherer, A. G./ Patzer, M. (Hrsg.): Betriebswirtschaftslehre und Unternehmensethik, Wiesbaden: Gabler, 103-124.

Bank für internationalen Zahlungsausgleich (2009): 79. Jahresbericht, Basel: BIZ.

Belke, A./ Gros, D. (2010): Global Liquidity, World Savings Glut and Global Policy Coordination, DIW Discussion Paper 973, Berlin: Deutsches Institut für Wirtschaftsforschung.

Binswanger, H. C. (2006): Die Wachstumsspirale. Geld, Energie und Imagination in der Dynamik des Marktprozesses, Marburg: Metropolis.

Bolton, P./ Thadden, E. L. von (1998): Blocks, Liquidity, and Corporate Control, in: The Journal of Finance, Vol. 53/No. 1, 1-25.

Borio, C./ Drehmann, M. (2009): Towards an Operational Framework for Financial Stability. 'Fuzzy' Measurement and its Consequences (BIS Working Papers 284), Basel: Bank für Internationalen Zahlungsausgleich.

Burghof, H. P./ Prothmann, P. (2008): Krisenbewältigung: Verpasste Chancen?, in: Wirtschaftsdienst, Jg. 88/Heft 11, 703-707.

Cheng, M./ Subramanyam, K. R./ Zhang, Y. (2005): Earnings Guidance and Managerial Myopia, Working Paper im Internet: http://papers.ssrn.com/sol3/Delivery.cfm/SSRN_ID851545_code388197.pdf?ab stractid=851545, Zugriff am 22.12.2009.

Chick, V. (1990): Some Methodological Issues in the Theory of Speculation, in: Moggridge, D. E. (Hrsg.): Keynes, Macroeconomics and Method (Perspectives on the History of Economic Thought IV), Aldershot: Edward Elgar, 113-124. 
Cohen, D. (2001): Unsere modernen Zeiten. Wie der Mensch die Zukunft überholt, Frankfurt/Main, NewYork: Campus.

Curbach, J. (2009): Die Corporate-Social-Responsibility-Bewegung, Wiesbaden: VS Verlag.

Dembinski, P. H. (2008): Finance Servante ou Finance Trompeuse, Rom, Paris: Edition Parole et Silence.

Deutsche Bundesbank (2009a): Ergebnisse der gesamtwirtschaftlichen Finanzierungsrechnung für Deutschland 1991 bis 2008, Juni 2009 (Statistische Sonderveröffentlichung 4), Frankfurt/Main: Deutsche Bundesbank.

Deutsche Bundesbank (2009b): Hochgerechnete Angaben aus Jahresabschlüssen deutscher Unternehmen von 1997 bis 2007, November 2009 (Statistische Sonderveröffentlichung 5), Frankfurt/Main: Deutsche Bundesbank.

Dewatripont, M./ Maskin, E. (1995): Credit and Efficiency in Centralized and Decentralized Economies, in: Review of Economic Studies, Vol. 62/No. 4, 541-555.

Elsas, R./ Krabnen, J. P. (1998): Is Relationship Lending Special? Evidence from Credit File Data in Germany, in: Journal of Banking and Finance, Vol. 22/No. 10-11, 1283-1316.

Emunds, B. (1995): Von einem Frosch, der kein Prinz ist. Oder: Warum ökonomische Rationalität nicht diskursethisch transformiert werden kann, in: Lesch, W./ Bondolfi, A. (Hrsg.): Theologische Ethik im Diskurs (UTB Wissenschaft 1806), Tübingen, Basel: Francke, 313-344.

Emunds, B. (2000): Finanzsystem und Konjunktur. Ein postkeynesianischer Ansatz, Marburg: Metropolis.

Emunds, B. (2008): Risiken, die niemand im Griff hat. Sozialethische Anmerkungen zur aktuellen Finanzmarktkrise, in: Herder-Korrespondenz. Monatshefte für Gesellschaft und Religion, Jg. 62/ Heft 9, 460-465.

Emunds, B. (2009a): Goodbye Wallstreet, Hello Wallstreet! Über den Bedarf, die kapitalmarktdominierte Finanzwirtschaft umzubiegen, in: Abmeier, K./ Dabrowski, M./ Wolf, J. (Hrsg.): Globalisierung und globale Gerechtigkeit, Paderborn: Schöningh, 55-83.

Emunds, B. (2009b): Die Krise der globalen Finanzwirtschaft - eine Analyse und sozialethische Einschätzung, in: Ethik und Gesellschaft 2009/2, im Internet: . http:/ /www.ethik-undgesellschaft.de/dynasite.cfm?dssid=5550\&dsmid=102026\#dstitle_836670, Zugriff am 2.7.2010.

Emunds, B. (2010): Art. Ökonomie/Wirtschaft, in: Enzyklopädie Philosophie, 2. Aufl., Bd. 2, Hamburg: Felix Meiner, 1846-1856.

Emunds, B./ Reichert, W. G. (2009): Finanzwirtschaft - kein Selbstzweck. Die Finanzkrise trifft vor allem die Schwellen- und Entwicklungsländer, in: Herder-Korrespondenz Jg. 63/Heft 5, 237-242.

Enderle, G. (1988): Wirtschaftsethik im Werden. Ansätze und Problembereich der Wirtschaftsethik, Stuttgart: Akademie der Diözese Rottenburg-Stuttgart.

Faust, M./ Kädtler, J. (2009): Shareholder Value - „die blödeste Idee der Welt“. Finanzkrise und Konzepte der Unternehmensführung, in: Mitteilungen aus dem SOFI, Jg. 3/Heft 6, 811.

Federal Reserve Board (2009): Federal Reserve Statistical Release. Flow of Funds Accounts of the United States. Historical Data, Release Date: September 17, 2009, im Internet: http://www.federalreserve.gov/Releases/z1/current/data.htm, Zugriff am 30. Oktober 2009.

Graham, J. R./ Harvey, C. R./ Rajgopal, S. (2005): The economic implications of corporate financial reporting, in: Journal of Accounting and Economics, Vol. 40/No. 1-3, 3-73. 
Hackethal, A./ Schmidt, R. H. (2000): Finanzsystem und Komplementarität, in: Franke, H. H./ Ketzel, E./ Kotz, H. H. (Hrsg.): Finanzmärkte im Umbruch, Berlin: Duncker \& Humblot, 53-102.

Hengsbach, F. (1991): Wirtschaftsethik. Aufbruch - Konflikte - Perspektiven, Freiburg/Br.: Herder.

Hengsbach, F. (1992): Wirtschaftsethik unter Druck und Gegendruck. Die Gewerkschaften, in: Koslowski, P. (Hg.): Neuere Entwicklungen in der Wirtschaftsethik und Wirtschaftsphilosophie, Berlin: Springer, 197-218.

Hengsbach, F. (1995): Abschied von der Konkurrenzgesellschaft. Für eine neue Ethik in Politik, Wirtschaft und Gesellschaft (Knaur-Taschenbuch 80073), München: Droermersche Verlagsanstalt Th. Knaur Nachf.

Hengsbach, F./ Möbring-Hesse, M. (2002): Verteilungspolitik im „Zeitalter des Arbeitsvermögens“. Vorschlag für ein gewerkschaftliches Politikprojekt, in: Gewerkschaftliche Monatshefte, Jg. 53/Heft 4-5, 232-242.

Homann, K. (2001): Ökonomik. Fortsetzung der Ethik mit anderen Mitteln, in: Siebeck, G. (Hrsg.): Artibus ingenuis. Beiträge zu Theologie, Philosophie, Jurisprudenz und Ökonomik, Tübingen: Mohr Siebeck, 85-110.

Homann, K./ Blome-Drees, F. (1992): Wirtschafts- und Unternehmensethik, Göttingen: Vandenhoeck \& Ruprecht.

Homann, K./ Lütge, C. (2005): Einführung in die Wirtschaftsethik, 2. Aufl., Münster: Lit.

Jensen, M. C. (1986): Agency costs of free cash flow, corporate finance, and takeovers, in: American Economic Review, Vol. 76/No. 2, 323-339.

Kädtler, J. (2009): Finanzialisierung und Finanzmarktrationalität. Zur Bedeutung konventioneller Handlungsorientierungen im gegenwärtigen Kapitalismus (SOFI Arbeitspapier 2009-5), Göttingen: Soziologisches Forschungsinstitut Göttingen an der GeorgAugust-Universität.

Kindleberger, C. P. (1996): Manias, panics, and crashes. A history of financial crises, 3. Aufl., New York: John Wiley.

Koslowski, P. (1997): Ethik der Banken und der Börse. Finanzinstitutionen, Finanzmärkte, Insider-Handel (Beiträge zur Ordnungstheorie und Ordnungspolitik 154), Tübingen: Mohr Siebeck.

Kubner, C. (2004): Unternehmensinteresse vs. Shareholder Value als Leitmaxime kapitalmarktorientierter Aktiengesellschaften, in: Zeitschrift für Unternehmens- und Gesellschaftsrecht, Jg. 33, 244-279.

Lane, C. (2000): Globalization and the German model of capitalism - erosion or survival?, in: British Journal of Sociology, Vol. 51/No. 2, 207-234.

Leggewie, C./ Welzer, H. (2009): Das Ende der Welt, wie wir sie kannten. Klima, Zukunft und die Chancen der Demokratie, Frankfurt/Main: S. Fischer.

Marx, K. (1964): Das Kapital, Band 3: Der Gesamtprozeß der kapitalistischen Produktion, (Marx Engels Werke 25), Berlin: Diez 1964 (11894).

Minsky, H. P. (1982): Can »it« happen again? Armonk/NY: Sharpe.

Minsky, H. P. (1986): Stabilizing an unstable economy, New Haven, London: Yale University Press.

Nastansky, A./ Strohe, H. G. (2009): Die Ursachen der Finanz- und Bankenkrise im Lichte der Statistik (Universität Potsdam - Wirtschafts- und Sozialwissenschaftliche Fakultät: Statistische Diskussionsbeträge 35), Potsdam.

Nell-Breuning, O. von (1954): An der Grenzscheide von Sozialpolitik und sozialer Strukturpolitik, München: Institut für Sozialpolitik und Arbeitsrecht. 
Nell-Breuning, O. von (1986): Kapitalismus - kritisch betrachtet. Zur Auseinandersetzung um das bessere „System“, 2. Aufl., Freiburg/Br.: Herder.

Ott, K./ Döring, R. (2008): Theorie und Praxis starker Nachhaltigkeit (Beiträge zur Theorie und Praxis starker Nachhaltigkeit), 2. Aufl., Marburg: Metropolis.

Pies, I. (2009): Unternehmensethik für die Marktwirtschaft. Moral als Produktionsfaktor, in: Ders.: Moral als Produktionsfaktor: Ordonomische Schriften zur Unternehmensethik, Berlin: wvb, 266-272.

Pies, I./ Winning, A. von/ Sardison, M./ Girlich, K. (2009): Nachhaltigkeit in der Mineralölindustrie. Theorie und Praxis freiwilliger Selbstverpflichtungen (Wirtschaftsethik-Studie 20091 des Lehrstuhls für Wirtschaftsethik der Martin-Luther-Universität Halle-Wittenberg), Halle: Lehrstuhl für Wirtschaftsethik.

Reichert, W. G. (2009): Das Ethos des Investmentbanking. Implikationen für eine prudentielle Regulierung, in: Ethik und Gesellschaft 2009/2, im Internet: http://www.ethik-undgesellschaft.de/dynasite.cfm?dssid=5550\&dsmid=102025\#dstitle_836668, Zugriff am 2.7.2010.

Richter, R./ Furubotn, E. G. (2003): Neue Institutionenökonomik. Eine Einführung und kritische Würdigung, 3. Aufl., Tübingen: Mohr Siebeck.

Sachverständigengruppe Weltwirtschaft und Sozialethik (2008): Verlagerung von Arbeitsplätzen. Entwicklungschancen und Menschenwürde. Sozialethische Überlegungen, Bonn: Sekretariat der Deutschen Bischofskonferenz.

Scherer, A. G. (2003): Multinationale Unternehmen und Globalisierung. Zur Neuorientierung der Theorie der multinationalen Unternehmung, Heidelberg: Physica-Verl.

Shiller, R. J. (2000): Irrationaler Überschwang. Warum eine lange Baisse an der Börse unvermeidlich ist, Frankfurt/Main, New York: Campus.

Sinn, H. W. (2009): Kasino-Kapitalismus. Wie es zur Finanzkrise kam, und was jetzt zu tun ist, Berlin: Econ.

Soskice, D. (1999): Globalisierung und institutionelle Divergenz. Die USA und Deutschland im Vergleich, in: Geschichte und Gesellschaft, Jg. 25/Heft 2, 201-225.

Steinmann, H./ Löhr, A. (1994): Grundlagen der Unternehmensethik, 2. Aufl., Stuttgart: Schäffer-Poeschel.

Streeck, $W$. (1992): Social institutions and economic performance. Industrial relations in capitalist economies, London: Sage Publications.

Streeck, W. (1999): Korporatismus in Deutschland. Zwischen Nationalstaat und Europäischer Union, Frankfurt/Main - New York: Campus.

Thielemann, U./ Breuer, M. (2000): Ethik zahlt sich langfristig aus - stimmt das?, in: Forum Wirtschaftsethik, Ausg. 2, 8-13.

Thielemann, U./Ulrich, P. (2003): Brennpunkt Bankenethik. Der Finanzplatz Schweiz in wirtschaftsethischer Perspektive, Bern: Haupt.

Ulrich, P. (1998): Integrative Wirtschaftsethik. Grundlagen einer lebensdienlichen Ökonomie, 2. Aufl., Bern: Haupt.

Wieland, J. (2005): Die Ethik der Governance, 4. Aufl., Marburg: Metropolis.

Windolf, P. (2008): Eigentümer ohne Risiko. Die Dienstklasse des Finanzmarktkapitalismus, in: Zeitschrift für Soziologie, Jg. 37/Heft 6, 516-535.

Windolf, P. (Hrsg.) (2005): Finanzmarkt-Kapitalismus. Analysen zum Wandel von Produktionsregimen, Wiesbaden: VS.

Windolf, P./ Beyer, J. (1995): Kooperativer Kapitalismus. Unternehmensverflechtungen im internationalen Vergleich, in: Kölner Zeitschrift für Soziologie und Sozialpsychologie, Jg. 47/Heft 1, 1-36. 